\title{
Assessment of the Two-Glutathione S-Transferase Genotypes GSTM1 and GSTT1 Polymorphism Frequencies in Epithelial Ovarian Cancer in Nasiriya Province
}

\author{
Enaas Saleh Jawad ${ }^{1}$, Hiba Abdul-Adheem² \\ 1Assist. Prof. Obstetrics and Gynecology Department, College of Medicine, University of Thi-Qar, Iraq, \\ ${ }^{2}$ Lecturer, Cancer Research Unit, College of Medicine, University of Thi-Qar, Iraq
}

\begin{abstract}
The family of glutathione S-transferases (GSTs) is composed of multiple isozymes with significant evidence of functional polymorphic variation. Glutathione S-transferase GSTM1 and GSTT1 are members of the GST family, is responsible for metabolism of xenobiotics and carcinogens. Allelic variant of GST gene polymorphisms might impair detoxification function and increase the susceptibility to cancer. The null genetic polymorphism in the gene encoding GST Class $\mu$, GSTM1 and GSTT1, has been reported to be significantly elevated in cancer patient. This study aimed to investigate if these polymorphisms are useful markers for predicting ovarian cancer susceptibility. 30 cases of epithelial ovarian cancer and 30 controls was used to investigate the frequency of GSTT1 and GSTM1 genotypes by using multiplex conventional PCR protocol. The frequency of GSTM1 null allele was significantly elevated $(100 \%, P=0.004)$ compared with $G S T T 1$ active allele $(13 \% P=0.037)$. GSTT1 null showed no significant effect in prognosis epithelial ovarian cancer $(17 \%, P=0.551)$. We conclude that the GSTs might affect in both risk to ovarian cancer.
\end{abstract}

Keywords: GSTM1, GSTT1 Polymorphism, Epithelial Ovarian Cancer, Nasiriya Province.

\section{Introduction}

Ovarian cancer is the main cause of death with gynecological malignancies ${ }^{[1]}$. It is a disease of premenopausal and postmenopausal women; however, no age exempt from ovarian neoplasm. Here is no socio- economic bar for ovarian malignancies but epidemiologically it is more frequent in higher socioeconomic and industrially developed countries ${ }^{[2]}$. Risk factor of ovarian carcinoma include inflammation, excessive number of lifetime ovulation, increase in steroid. Hormone levels, infertility, age, asbestos, talk and reproductive factor such as nulli-parity ${ }^{[3]}$. Oxidative stress is due to disturbance in the balance between the production of reactive oxygen species (ROS) and deficiency of antioxidant defense. In other words, oxidative stress results if excessive production of ROS overwhelms the antioxidant system or if there is a significant decrease or lack of antioxidant defense [4]. Numerous genetic polymorphism that may be involved in deferential enzyme function or expression, cancer risk $^{[5]}$. Our study postulated that lack of detoxification, which is determined genetically, might be risk factor of ovarian cancer. Glutathione S-transferase (GSTs) are a family of enzymes responsible for the metabolism of xenobiotics and carcinogens. GSTM1, one member of GST family was formerly termed GST1 or GST class "mu"[6]. GSTM1 is critical in the detoxification of the oxidative stress product during ovulation ${ }^{[7]}$. Because of detoxification properties of GST enzymes, it is logical to suspect the role of GSTM1-related gene in ovarian cancer patients ${ }^{[8]}$. Glutathione S-transferase theta 1 (GSTT1) is located in long arm of chromosome 22 (22q11.23). Some studies reported that GSTT1 protein was involved in the conjugation of ethylene oxide and halogenated metabolites ${ }^{[10]}$. Glutathione S-transferase catalyze glutathione-mediated reduction of exogenous and endogenous electrophile with broad and overlapping substrate specificity ${ }^{[11]}$. It has been hypnotized GST functional variant associated with less effect detoxification of potential carcinogenesis may confer an increased susceptibility to cancer. Especially in the presence of environmental stress. Such as smoking 
and exposure UV radiation ${ }^{[12]}$. Detection variant of null allele exist for GSTT1 and GSTM1 genes and these present biochemically as failure express protein ${ }^{[13,14]}$.

Reported studies about the effect of GST polymorphism on risk of ovarian cancer are still few, due to many limitations. GSTT1 and GSTM1 null genotypes was shown to be associated with proper survival and progression free interval in ovarian cancer cases [15]. This study has been done because of the lack of genetic studies related to these cases in south of Iraq.

\section{Materials and Method}

Subjects: This case-control study was conducted between February 2016 and December 2017. Overall, thirty cases who recruited at Al-Habbobi teaching hospital were histopathological confirmed as epithelial ovarian cancer after surgery. Thirty healthy volunteers were chosen as control. Age ranged from 22 to 39 years in epithelial ovarian group and from 21 to 49 years in control group. Peripheral blood ( $5 \mathrm{~mL}$ ) was drawn and collected into tubes containing ethylenediaminetetraacetic acid) EDTA) as an anticoagulant. The collected blood samples were frozen until DNA extraction and then PCR analysis was proceeded. A verbal consent had been taken from the patient and the control volunteer before engagement with the questionnaire.

Genotyping: Genomic DNA was isolated from whole blood as described previously ${ }^{[16]}$. Eight microliter of DNA of each sample was subjected to qualitative assay by $0.8 \%$ agarose gel electrophoresis $(70 \mathrm{~V} / 150 \mathrm{Ma}$ for $20 \mathrm{~min}$ ). The homozygous of GSTT1 and GSTM1 genes were detected by multiplex PCR reaction, using primer set sequences in the table 1. To check successful amplification, albumin gene was used as internal control.
The absence of amplified GSTT1 or GSTM1 product (in the existence of the albumin PCR product) indicate the null genotyping for each respectively. Multiplex PCR reaction was proceed in a reaction volume $20 \mu \mathrm{l}$ which contain $5 \mu \mathrm{l}$ of master mix (Kappa, Korea), 1.25 $\mu l$ of each primer (10 pmol), $3.5 \mu l$ of genomic DNA. Amplification condition for this multiplex reaction was as follows : $\left(94^{\circ} \mathrm{C}\right.$ for $3 \mathrm{~min}, 30$ cycles at $94^{\circ} \mathrm{C}$ for $1 \mathrm{~min}$, $58^{\circ} \mathrm{C}$ for $1 \mathrm{~min}$ and $72^{\circ} \mathrm{C}$ for $1 \mathrm{~min}$, and final cycle of extension at $72^{\circ} \mathrm{C}$ for $5 \mathrm{~min}$ ). $10 \mu \mathrm{l}$ of each PCR product was loaded into $1.8 \%$ agarose gel containing ethidium bromide for electrophoresis $(70 \mathrm{~V}, 150 \mathrm{~mA}$ for $30 \mathrm{~min})$ and visualized at room temperature under UV light.

Epidemiological Analysis: Relative association between studied genotypes and epithelial ovarian cancer risk were evaluated to calculate odd ratio (OR) and 95\% confidence intervals (CI).

\section{Results}

Genomic DNA, which was extracted from blood leukocytes, was subjected to agarose gel electrophoresis assay as previously described, was illustrated in figure 1 . Thirty samples of ovarian cancer and thirty samples of healthy volunteers (as control) genotyped by multiplex PCR assay for detection of GSTT1 and GSTM1 genes. Figure 2 represent multiplex PCR products which was analyzed by $1.8 \%$ agarose gel electrophoresis. The GSTT1 active genotype frequency was $43.3 \%$ which was significantly different from control $(P=0.037$, OR 2.75, 95\%), whereas the GSTM1 null genotype frequency was $100 \%$ for all ovarian cancer cases $(P=0.004$, OR 0.02 , 95\%). GSTT1 null genotype showed no significant effect in risk of epithelial cancer $(P=0.551$, OR $1.3,95)$, as illustrated in table 1 .

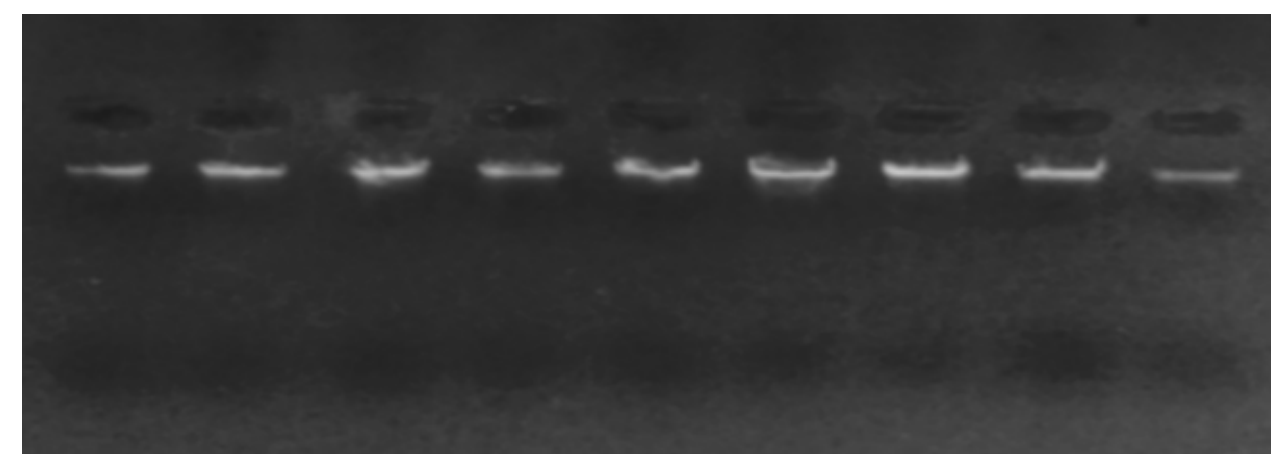

Figure 1. Extracted genomic DNA analyzed by $0.8 \%$ agarose gel electrophoresis 


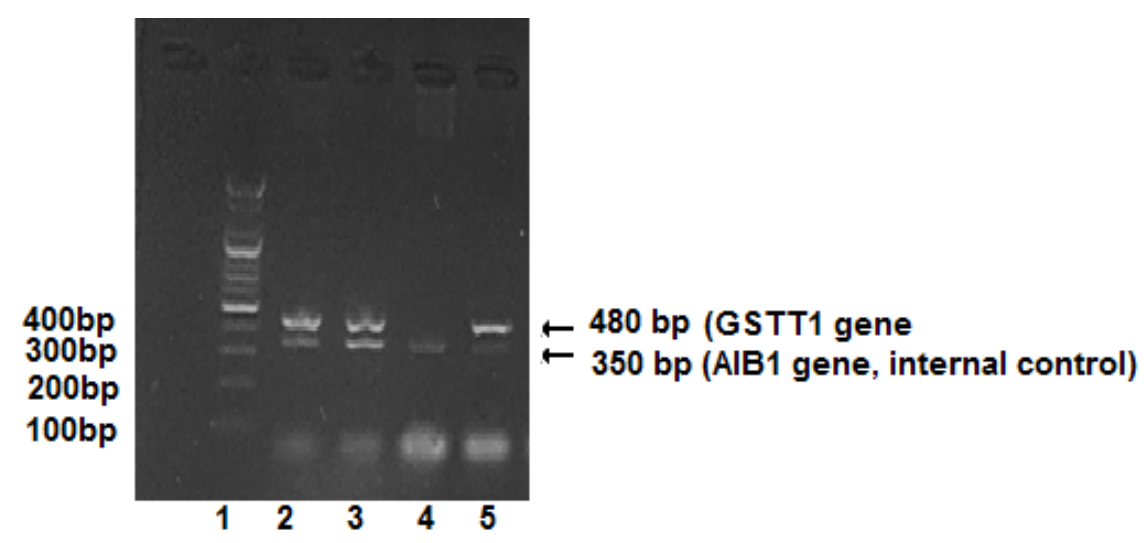

Figure 2. Detection of GSTT1 and GSTM1 genotypes by multiplex conventional PCR. Arrows indicate the position of $480 \mathrm{bp} G S T T 1$ product and $350 \mathrm{bp} A I B 1$ product. lane 1 is a $100 \mathrm{bp}$ marker. Lane 2.3. 5 refer to GSTT1 product, lane 4 was classified as GSTT1 null. All lane exhibit GSTM1 null at 215bp.

Table 1: Null frequency in ovarian cancer

\begin{tabular}{|l|c|c|c|c|}
\hline & Total & & $\boldsymbol{P}_{\text {value }}{ }^{\text {a }}$ & OR (95\% CI) $^{\mathbf{b}}$ \\
\hline Control & 30 & & & \\
\hline GSTT1 active & 30 & $13(43.3 \%)$ & 0.037 & $2.75(1.06-7.17)$ \\
\hline GSTT1 null & 30 & $17(56.6 \%)$ & 0.551 & $1.3(0.541-3.15)$ \\
\hline GSTM1 null & 30 & $30(100 \%)$ & 0.004 & $0.02(0.001-0.28)$ \\
\hline
\end{tabular}

a. Fisher exact test, b. Odd ratio with $95 \%$ confidence interval parentheses

Other risk factor had been assessed to be considered as non-significant risk factor

\section{Discussion}

This is the first study to investigate the association between GSTT1 and GSTM1 polymorphisms and the epithelial ovarian cancer in Nasiriya city. This study reported that GTT1 active and GSTM1 null have significant effects in risk of epithelial ovarian cancer. Except the genotype GSTT1 null showed no significant effect of any examined cases. In the past several years, many studies have tried to illustrate the effects of genetic factors in susceptibility and development of ovarian cancer. However, the reported studies about frequencies mutation of previously mentioned genes were mostly not exhaustive ${ }^{[17,18]}$. Recently, many studies reported that the polymorphism of GSTs could effect in susceptibility and developing ovarian cancer due to their important role in modification of the biological effects of carcinogens $^{[19,20]}$.

Deletion of GSTM1 and GSTT1 genes results in "null" genotype characterized by a general deficit in enzymatic activity ${ }^{16}$. Individuals homozygous of these deletions are thought to be at increased risk for malignancies as consequence of a decreased capacity to detoxify possible carcinogens ${ }^{[21]}$. GSTM1 gene deletion is a good marker for the early detection of many diseases, including, ovarian cancer, endometriosis ${ }^{[7]}$, cystic fibrosis $^{[22]}$, bladder $^{[23]}$, lung $^{[24]}$ and stomach ${ }^{[25]}$ cancers. Null phenotype of GSTT1 exhibits decrease in catalytic activity and has been associated with increased risk of cancer of the head, neck and oral cavity ${ }^{[26]}$. Patients with GSTM1 null or GSTT1 null showed a better survival rate after chemotherapeutic treatment for the invasive ovarian cancer compared to other patient ${ }^{[27] .}$

Otherwise, this study found that the risk of ovarian cancer was increased in carrier of GSTT1-active genotype. This result seemed to be conflict, taking into consideration that several environmental carcinogens found in combustion products and tobacco smoke are among GSTT1 substrates ${ }^{[20]}$. However, there is proof that GSTT1 might also be involved in bioactivation, rather than detoxification of several bifunctional alkylating 
agents, present in environmental pollution and certain occupational hazards ${ }^{[28]}$. As a conclusion, bioactive process can yield potent electrophiles that modify DNA and are potentially genotoxic ${ }^{[29,30]}$. Thus, out result in conformity with the study of Sgambato et al. they showed that GSTT1 null is associated with decreased risk of cancer ${ }^{[31]}$.

In summary, these results suggest that GSTM1 null and GSTT1 active alleles increase risk to ovarian cancer in Nasiriya population, but further large sample studies need to confirm the genetic role of GSTs in epithelial ovarian cancer.

Financial Disclosure: There is no financial disclosure.

Conflict of Interest: None to declare.

Ethical Clearance: All experimental protocols were approved under the Obstetrics and gynecology department and all experiments were carried out in accordance with approved guidelines.

\section{References}

1. Ferlay J, Pisani P, Soerjomataram I. Cancer incidence and mortality worldwide: sources method and major patterns in GLOBOCAN 2012. Int. J. Cancer. 2015; 359-386.

2. Padubidri VG, Daftary SN. Ovarian cancer. Shaws Textbook of Gynecology 2015, 16th edition, p 521.

3. Dubeau L. The cell of origin of ovarian epithelial tumors. Lancet Oncol 2008; 9(12): 1191-1197.

4. Kang DH, oxidative stress, DNA damage and breast cancer. AACN Clin issue 2002; 13: 540-549.

5. Agarwal G, Tulsyan S, Lal P, Mittal B. Generalized Multifactor Dimensionality Reduction (GMDR) Analysis of Drug-Metabolizing Enzyme-Encoding Gene Polymorphisms may Predict Treatment Outcomes in Indian Breast Cancer Patients. World Journal of Surgery 2016; 40(7): 1600-1610.

6. Board PG, Menon D. Glutathione transferases, regulators of cellular metabolism and physiology. BBA 2013; 1830(5):3267-3288.

7. Baxter SW, Thomas EJ and Compbell IG. GSTM1 null polymorphism and susceptibility to endometriosis and ovarian cancer. Carcinogenesis 2001; 22: 63-65.

8. Hsieh YY, Chang CC, Tsai FJ, Lin CC, Chen $\mathrm{JM}$, Tsai $\mathrm{CH}$. Glutathione $\mathrm{S}$ transferase $\mathrm{M} 1$ * null genotype but not myleoperoxide promoter G-463A polymorphism is associated with higher susceptibility to endometriosis. Molecular Human Reproduction 2004; 10(10): 713-717.

9. Han J, Deng W, Wang L, Qi W. Null genotypes of glutathione S-transferase $\mu 1$ and glutathione S-transferase $\theta 1$ are associated with osteosarcoma risk: A meta-analysis. Oncology Letters 2015; 9: 1912-1916.

10. Minelli C, Wei I, Sagoo G. Interactive effects of antioxidant genes and air pollution on respiratory function and airway disease. AHuGE review. AM J Epidemiol 2011;173:603-620.

11. To-Figueras J, Gené M. Microsomal epoxide hydrolase and glutathione S-transferase polymorphisms in relation to laryngeal carcinoma risk. Cancer Letters 2002; 1(2): 95-101.

12. Sharma A, Gupta S. Glutathione S-transferase M1 and T1 polymorphisms, cigarette smoking and HPV infection in precancerous and cancerous lesions of the uterine cervix. Asian Pacific Journal of Cancer Prevention 2015; 16(15): 6429-6438.

13. De Sá RA, Moreira AS. Human glutathione S-transferase polymorphisms associated with prostate cancer in the Brazilian population. IntBraz J Urol. 2014; 40: 463-73

14. Hayes JD. Strange RC. Glutathione S-transferase polymorphisms and their biological consequences. Pharmacology 2000;61:154-166.

15. Lo H, Ali-Osman F. Genetic polymorphism and function of glutathione S-transferases in tumor drug resistance. Current Opinion in Pharmacology 2007; 7(4): 367-374.

16. Beeghly A, Katsaros D. Glutathione S-transferase polymorphisms and ovarian cancer treatment and survival. Gynecologic Oncology 2006; 100(2): 330-337.

17. Abbas AT, Al-amery AN, Abdul-Adheem HA. Investigation of eNOS gene intron $4 \mathrm{~A} / \mathrm{B}$ VNTR polymorphism in patient with essential hypertension. International Journal of Research in Applied 2016; 4(2):85-92.

18. Desai A, Xu J, Aysola K. Epithelial ovarian cancer: An overview. World J Transl Med. 2014; 3:1-8.

19. Krivokuca A, Dobricic J. BRCA negative individuals from Serbian hereditary breast and ovarian cancer families. J BOUN 2013; 594-600. 
20. $\mathrm{Wu} \mathrm{B}$, Dong D. Human cytosolic glutathione transferases: structure, function and drug discovery. Trend Pharmacol Sci. 2012; 656-686.

21. Xu C, Chen S, Wang T. Quantitative assessment of the influence of glutathione transferase M1 null variant on ovarian cancer risk. J Cancer Res Ther 2014; 10:201-205.

22. Marson FA, Bertuzzo CS. Polymorphisms in the glutathione pathway modulate cystic fibrosis severity: a cross-sectional study .BMC Medical Genetics 2014; 15(27): 1471-2350.

23. Marson FA, Bertuzzo CS. Genetic interaction of GSH metabolic pathway genes in cystic fibrosis. BMC Medical Genetics 2013; 14:60.

24. Gracia-Closas M, Malats N, Silverman D. NAT2 slow acetylation and GSTM1 null genotypes increase bladder cancer risk: result from Spanish Bladder Cancer Study and meta-analysis. Lancet 2005; 366(9486): 649-659.

25. Yang Z, Qui M, Hu J. GSTM1 null genotype contributes to lung cancer risk in Asian population: A meta-analysis of 23 studies. PLOS ONE 2013; 8(4): e 62181.

26. Zhang X, Cui Y. GSTM1 null genotype and gastric cancer risk in the Chinese population: An update meta-analysis and review. Dove Press J 2015:8: 969-975.

27. Coles BF, Kadlubar FF. Human Alpha Class Glutathione S-Transferases: Genetic Polymorphism, Expression, and Susceptibility to Disease. Method in Enzymology 2005;401: 42-49.

28. Howells RE, Holland T, Dhar KK. Glutathione S-transferase GSTM1 and GSTT1 genotypes in ovarian cancer: association p53 expression and survival. Int J Gynecol Cancer 2001; 11(2): 107112.

29. Loecken EM, Dasari S. The bis-electrophile diepoxybutane cross-links DNA to human histones but does not result in enhanced mutagenesis in recombinant systems. NIH Public Access 2009; 22(6): 1069-1076.

30. Akiba N, Shiizaki K. Influence of GSH S-transferase on the mutagenicity induced by dichloromethane and 1, 2-dichloropropane. Mutagenesis 2017; 32(4): 455-462.

31. Sgambato A, Campisi B, Zuba A. Glutathione S-transferase (GST) polymorphism as risk factors of cancer in a highly homogeneous population from southern Italy. Anticancer Res 2002; 22(6B): $3647-$ 365. 